\title{
Metabolic regulation of magnolol on the nuclear receptor, liver $\mathrm{X}$ receptor
}

\author{
NA XIE ${ }^{1}$, CHUNYANG HU $^{1}$, ANRAN GUO $^{2}$, HAO LIANG $^{2}$, PENGCHENG DU $^{1}$ and GUOTIAN YIN ${ }^{1}$ \\ ${ }^{1}$ Department of Cardiology, The Third Affiliated Hospital of Xinxiang Medical University, Xinxiang, Henan 453000; \\ ${ }^{2}$ Department of Internal Medicine, Luyi Xian People's Hospital, Zhoukou, Henan 477200, P.R. China
}

Received May 27, 2014; Accepted January 29, 2015

DOI: $10.3892 / \mathrm{etm} .2015 .2300$

\begin{abstract}
The aim of the present study was to investigate whether magnolol, the essential component of the traditional Chinese medicine, Magnolia officinalis, can pass through liver $\mathrm{X}$ receptor $\alpha(\mathrm{LXR} \alpha)$, to subsequently play an important role in the lipid metabolic balance. Using a HepG2 human hepatoma cell line, mammalian cellular one-hybridization and mammalian cell transcriptional activation experiments were performed to detect the combination degree of magnolol at different concentrations with $\mathrm{LXR} \alpha$, and assess the transcriptional activity. In addition, using a THP-1 human monocytic cell line, quantitative polymerase chain reaction was performed to assess the effect on the expression levels of downstream genes. Magnolol was shown to dose-dependently combine with LXR $\alpha$, and subsequently regulate the transcriptional activity of LXR $\alpha$. In addition, magnolol was found to adjust the expression of associated $\operatorname{LXR} \alpha$ downstream genes in the macrophages. In conclusion, magnolol was demonstrated to affect LXR $\alpha$, which may outline a new molecular mechanism through which magnolol exerts a lipid-lowering function.
\end{abstract}

\section{Introduction}

Due to the growth and improvement in living standards, the changes in eating habits and lifestyles and the increasingly aging population, the incidence of metabolic diseases, such as diabetes and atherosclerosis (AS), is increasing each year. According to statistics from the World Health Organization, ischemic heart disease ranks first among the top ten fatal diseases in 2011 (1). Ischemic heart disease is pathologically and largely based on AS, and the majority of AS cases are caused by a metabolic disturbance in glucolipids. Previous

Correspondence to: Dr Pengcheng Du and Dr Guotian Yin, Department of Cardiology, The Third Affiliated Hospital of Xinxiang Medical University, 601 East Hualan Road, Xinxiang, Henan 453000, P.R. China

E-mail: pengchengducn@163.com

E-mail: guotianyin@yeah.net

Key words: Magnolia officinalis, magnolol, liver $\mathrm{X}$ receptor $\alpha$, cholesterol studies have shown that nuclear receptors (NRs) are closely associated with glucolipid metabolism in vivo $(2,3)$. Liver $\mathrm{X}$ receptor (LXR), as an important member of the NR superfamily, participates in the regulatory processes of a number of physical activities in vivo, including the metabolism of cholesterol, glucose and fat, inflammation and the maintenance of the metabolic balance. LXR has attracted increasing attention due to its important function in glucolipid metabolism (4). There are two subtypes of LXR: LXR $\alpha$ and LXR $\beta$. The expression of LXR $\alpha$ is tissue-specific, with $\mathrm{LXR} \alpha$ highly expressed in the liver, intestine, kidney, adrenal gland, spleen, adipose tissue and macrophages, while LXR $\beta$ is extensively expressed in tissue cells. As previously demonstrated, application of a LXR agonist can reduce the intracellular cholesterol content by decreasing the rate of intestinal cholesterol absorption, increasing the level of bile acid and decreasing the rate of reverse cholesterol transfer, thereby ameliorating AS $(5,6)$.

Due to their structural diversity, small natural product molecules have become an essential source of small molecular lead compounds. Magnolol is one of the key monomer components of the traditional Chinese medicine, Magnolia officinalis extract (7). Magnolia officinalis has a long history as a traditional Chinese medicine and is largely applied for dispelling a cold, relieving headaches, inhibiting anxiety, treating diarrhea and apoplexia, removing chest stuffiness, composing central nerves, and as an anti-fungus and anti-ulcer treatment (8-10). In addition, Jiangzhi Ninggan capsules, which exhibit lipid-lowering effects, contain magnolol (11). Magnolol has been demonstrated to interact with the retinoid $\mathrm{X}$ receptor $\alpha$ $(\mathrm{RXR} \alpha)$ and the peroxisome proliferator-activated receptor $\gamma$, amongst other NRs associated with glucolipid metabolism (12). Therefore, in the present study, whether magnolol interacts with the LXR to regulate LXR-targeted expression of downstream genes was investigated. The aim of the study was to provide further understanding into the lipid-lowering mechanism of magnolol, in order to offer a novel idea for the research and development of anti-AS drugs.

\section{Materials and methods}

Cell culture. All the cells were cultured in an environment with $5 \% \mathrm{CO}_{2}$ at $37^{\circ} \mathrm{C}$. The HepG2 human hepatoma cell line (American Type Culture Collection, Manassas, VA, USA) was cultured in Minimal Essential Medium (MEM; Gibco 
Life Technologies, Grand Island, NY, USA), supplemented with $10 \%$ fetal bovine serum (FBS; Gibco Life Technologies), $100 \mathrm{U} / \mathrm{ml}$ penicillin and $100 \mu \mathrm{g} / \mathrm{ml}$ streptomycin (Sigma-Aldrich, St. Louis, MO, USA). The THP-1 human monocyte cell line (American Type Culture Collection) was cultured in RPMI-1640 medium (Gibco Life Technologies) containing 10\% FBS with $10 \mathrm{mmol} / 1 \mathrm{HEPES}, 100 \mathrm{U} / \mathrm{ml}$ penicillin and $100 \mu \mathrm{g} / \mathrm{ml}$ streptomycin (Sigma-Aldrich).

Transient cellular transfection and luciferase reporter gene assay. Following HepG2 cell growth to a density of 30-50\%, the cells were seeded in a 24-well plate with non-serum MEM. Lipofectamine 2000 transfection reagent (Invitrogen Life Technologies, Carlsbad, CA, USA) was applied for the transfection of the plasmids into the cells. The mammalian one-hybridization experiment is a method extensively applied to identify transcription factors at a molecular level. As an agonist of LXR, T0901317 (J\&K Scientific Ltd., Shanghai, China) is known to effectively activate the transcriptional activity of the LXR $\alpha$-ligand binding domain (LBD) (13). Using the mammalian one-hybridization method and T0901317 as a positive control, the effect of various concentrations of magnolol (J\&K Scientific Ltd.) on the transcriptional activity of the LXR $\alpha$-LBD was analyzed in order to identify whether magnolol was able to directly combine with the LXR $\alpha$-LBD.

In the mammalian one-hybrization experiment, $400 \mathrm{ng}$ pCMX-Gal4-DBD-LXR $\alpha$-LBD, 400 ng UAS-TK-Luc, and $100 \mathrm{ng}$ reference pRL-SV40 plasmid were transfected (Promega Corporation, Madison, WI, USA). In the transcriptional activation experiment, $400 \mathrm{ng}$ pcDNA3.1a-LXR $\alpha$, 400 ng pcDNA3.1a-RXR $\alpha, 400$ ng pGL2-basic-LXRE-Luc plasmids and $100 \mathrm{ng}$ reference plasmid pRL-SV40 were transfected (Promega Corporation). pRL-SV40 was used as the negative control. After cellular transfection for $6 \mathrm{~h}$, the medium was changed to complete medium, 10,20 or $40 \mu \mathrm{M}$ magnolol was added and incubated for $18-24 \mathrm{~h}$. The positive control received $50 \mathrm{nM}$ T0901317 and the negative control contained $10 \mu \mathrm{M}$ DMSO. Following incubation, the cells were washed with phosphate-buffered saline (PBS), and $100 \mu 1$ lysate was added to each well for cell lysis at $37^{\circ} \mathrm{C}$ for $20 \mathrm{~min}$. Subsequently, the activity of the firefly luciferase and reference luciferase were detected, according to the manufacturer's instructions (Promega Corporation, Madison, WI, USA).

Quantitative polymerase chain reaction (PCR). Prior to the PCR experiment, $160 \mathrm{nmol} / \mathrm{l}$ phorbol ester (Sigma-Aldrich) was incubated with the THP-1 cells for $24 \mathrm{~h}$ to induce cell differentiation into macrophages. The cells were subsequently cultured in non-serum medium containing $50 \mu \mathrm{g} / \mathrm{ml}$ oxidized low-density lipoprotein (Jingmei Biotechnology Co., Nanjing, China) for $48 \mathrm{~h}$, and lipids were phagocyted, then formed foam cells. After $24 \mathrm{~h}$ of treatment, the medium in the Petri dish was removed and the cells were washed with PBS. The total RNA was extracted using TRIzol reagent (Takara Biotechnology Co., Ltd., Dalian, China), according to the manufacturer's instructions. A reverse transcription kit (Takara Biotechnology Co., Ltd.) was used to convert the extracted RNA into cDNA, after which quantitative PCR detection was conducted. The primer sequences were as follows: Human ATP-binding cassette transporter A1 (ABCA1) forward, 5'-GATTGGCTT CAGGATGTCCATGTTGGAA-3' and reverse, 5'-GTATTT TTGCAAGGCTACCAGTTACATTTGACAA-3'; human ATP binding cassette transporter G1 (ABCG1) forward, GCCACTTTCGTGGGCCCAGTGA-3' and reverse, 5'-TCT CATCACCAGCTGTGTTGCA-3'. $\beta$-Actin was used as the reference gene in the genetic expression experiments, and the $\beta$-actin primers were as follows: Forward, 5'-GCGGGAAAT CGTGCGTGAC-3' and reverse, 5'-CGTCATACTCCTGCT TGCTG-3'.

Sample cDNA underwent quantitative PCR amplification using a SYBR fluorescent probe kit (Takara Biotechnology Co., Ltd.). The amplification procedure was as follows: Initial denaturation at $95^{\circ} \mathrm{C}$ for $10 \mathrm{sec}$, followed by 40 cycles of $95^{\circ} \mathrm{C}$ for $5 \mathrm{sec}, 60^{\circ} \mathrm{C}$ for $20 \mathrm{sec}, 72^{\circ} \mathrm{C}$ for $10 \mathrm{sec}$ and a final extension at $72^{\circ} \mathrm{C}$ for $10 \mathrm{~min}$.

Statistical analysis. All experimental data are expressed as the mean \pm standard deviation. Statistical analysis was performed using GraphPad Prism software (GraphPad Software, Inc., La Jolla, CA, USA). The statistical significance of differences was analyzed by one way analysis of variance and Dunnett's post hoc test, where $\mathrm{P}<0.05$ was considered to indicate a statistically significant difference.

\section{Results}

Magnolol is an agonist of LXR $\alpha$. At $6 \mathrm{~h}$ after HepG 2 cell transfection with the corresponding plasmid, magnolol was applied at different concentrations, as well as T0901317. After 18-24 h, the detection of luciferase activity was performed. T0901317 was shown to markedly stimulate the transcriptional activity of the LXR $\alpha$-LBD, while magnolol was shown to dose-dependently increase luciferase activity. These results indicated that magnolol was able to interact with the LXR $\alpha$-LBD directly (Fig. 1). NRs are ligand-dependent transcription factors, and magnolol was shown to combine with the LXR $\alpha$-LBD at a molecular scale, demonstrating that magnolol may affect the expression of LXR downstream genes by combining with LXR response elements (LXRE).

Magnolol increases the transcriptional activation of $L X R \alpha$. To function as transcription factor, LXR is required to form a heterodimer with the RXR, with the combination in the LXRE, in order to initiate downstream genetic expression. A mammalian transcriptional activation experiment was conducted to detect whether magnolol was able to promote the combination of the LXR/RXR heterodimer and LXRE. At $6 \mathrm{~h}$ after HepG2 cell transfection with the corresponding plasmids, the cells were treated with magnolol at various concentrations or T0901317 (positive control) for 24 h. As shown in Fig. 2, T0901317 significantly increased the luciferase activity, promoted the combination of the LXR/RXR heterodimer and LXRE, and increased the transcriptional activity of the LXRE. Furthermore, magnolol was shown to dose-dependently increase the transcriptional activity of LXRE. These results indicated that magnolol was able to regulate the combination of the LXR/RXR heterodimer and LXRE, thereby affecting the expression of LXR downstream genes. 


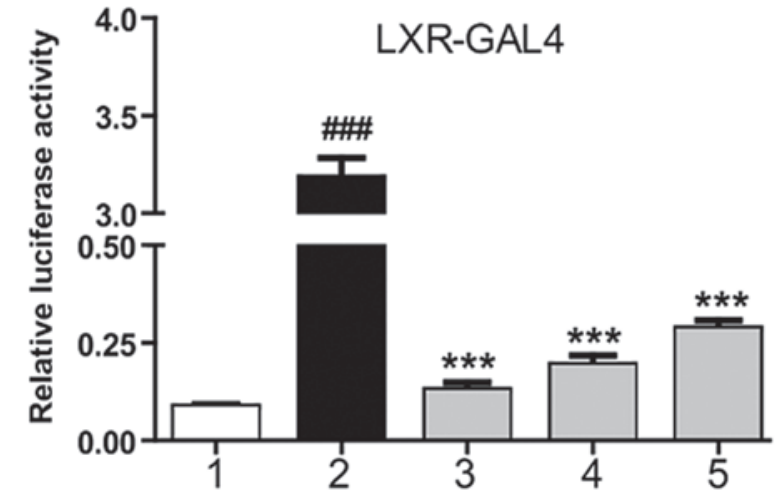

Figure 1. Effect of magnolol on LXR-GAL4 one-hybridization activity. 1, dimethyl sulfoxide negative control group; 2, T0901317 (50 nM) positive control group; 3 , low-dose magnolol group $(10 \mu \mathrm{M}), 4$, medium-dose magnolol group $(20 \mu \mathrm{M})$; 5, high-dose magnolol group $(40 \mu \mathrm{M})$. ${ }^{\# \# \#} \mathrm{P}<0.001$, vs. negative control groups; ${ }^{* * *} \mathrm{P}<0.05$, vs. negative control group. LXR, liver $\mathrm{X}$ receptor.

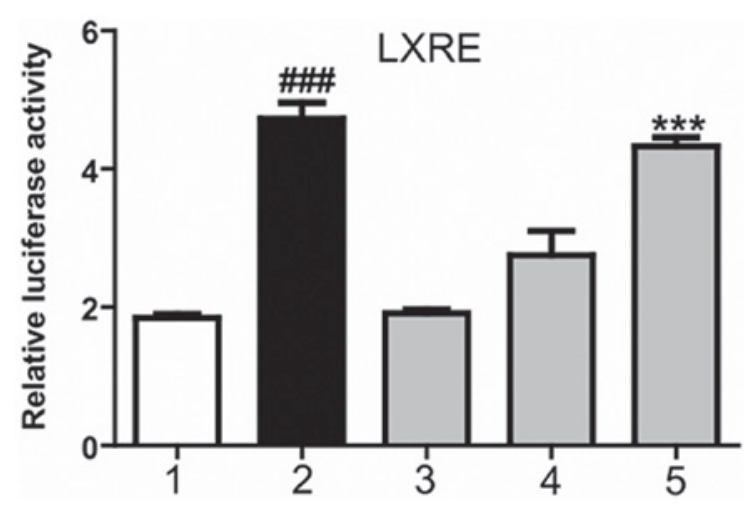

Figure 2. Effect of magnolol on LXRE transcriptional activity. 1, dimethyl sulfoxide negative control group; 2, T0901317 $(1 \mu \mathrm{M})$ positive control group; 3 , low-dose magnolol group $(10 \mu \mathrm{M}), 4$, medium-dose magnolol group $(20 \mu \mathrm{M}) ; 5$, high-dose magnolol group $(40 \mu \mathrm{M}) .{ }^{\# \# \#} \mathrm{P}<0.001$, vs. negative control groups; ${ }^{* * *} \mathrm{P}<0.001$, vs. negative control group. LXRE, liver $\mathrm{X}$ receptor response elements.

Magnolol increases the expression levels of the LXR $\alpha$ downstream genes, $A B C A 1$ and $A B C G 1$. ABCA1 and ABCG1 are two essential proteins that mediate cholesterol efflux in macrophages (14). In order to investigate whether magnolol affects the expression of ABCA1 and ABCG1 via the LXR, and subsequently ameliorates AS, the effect of magnolol on the expression of ABCA1 and ABCG1 was investigated in THP-1 macrophage-derived foam cells using quantitative PCR. Magnolol was applied at various concentrations to treat the cells. Magnolol was demonstrated to markedly increase the mRNA expression levels of ABCA1 and ABCG1, and the effect became more significant as the magnolol concentration increased (Fig. 3). Therefore, the results indicated that magnolol dose-dependently regulated the mRNA expression levels of ABCA1 and ABCG1.

\section{Discussion}

In clinical practice, as a result of good pharmacological effects, magnolol is widely applied in drugs with Magnolia officinalis as the major component, such as Huoxiang Zhengqi
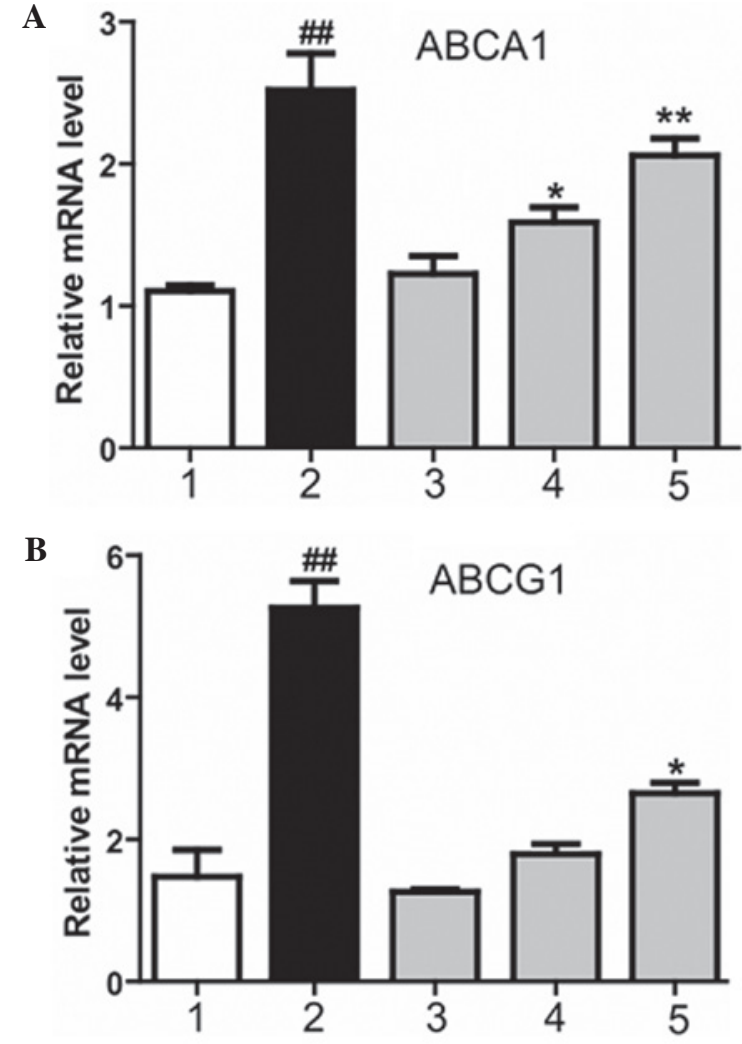

Figure 3. Effect of magnolol on the mRNA expression levels of (A) ABCA1 and (B) ABCG1, downstream genes of the liver X receptor. 1, dimethyl sulfoxide negative control group; 2, T0901317 (2 $\mu \mathrm{M})$ positive control group; 3, low-dose magnolol group $(10 \mu \mathrm{M}), 4$, medium-dose magnolol group $(20 \mu \mathrm{M})$; 5 , high-dose magnolol group $(40 \mu \mathrm{M}) .{ }^{\# \#} \mathrm{P}<0.01$, vs. negative control group; ${ }^{* *} \mathrm{P}<0.01$ and ${ }^{*} \mathrm{P}<0.05$, vs. negative control group. ABCA1, ATP-binding cassette transporter A1; ABCG1, ATP binding cassette transporter G1.

Shui, Banxia Houpo Tang, Dachengqi Tang, Tongfusan and Jiangzhining liver capsules. Numerous studies have investigated the pharmacological effects of magnolol, of which a number have been demonstrated, including the inhibition of inflammation to protect endothelial cells from damage, relieving acute inflammatory pain, inhibiting cellular mutation by suppressing mutant enzyme activity, and increasing the combination degree of tranquilizers and the $\gamma$-aminobutyric acid (GABA) receptor by increasing the expression of the GABA receptor; thus, magnolol ultimately achieves central sedative and anxiolytic effects (14-16). In addition, magnolol has been shown to inhibit skin photoaging by constraining the nuclear transcription factor, nuclear factor- $\kappa \mathrm{B}$ (17). However, there are comparatively less studies investigating the lipid-lowering mechanism of magnolol. One of the research areas in AS is the regulatory systems of LXR $\alpha$; thus, the present study hypothesized that magnolol interacts with LXR $\alpha$ to aid lipid-lowering.

In the present study, using a HepG2 cell line, magnolol was shown to directly combine with the NR, LXR $\alpha$-LBD, and increase LXR transcriptional activation. In addition, using a THP-1 cell line, magnolol was demonstrated to regulate the expression of ABCA1 and ABCG1, which are downstream genes of LXR. Previous studies have indicated that the expression of ABCA1, ABCG1 and ABCG5/8, LXR downstream genes, can affect the formation of high-density lipoprotein 
(HDL) (18-21), while the latest treatment method for AS is to increase the level of HDL cholesterol in the serum $(22,23)$. Therefore, the results of the present study indicate that magnolol may promote cholesterol efflux by increasing the expression levels of ABCA1 and ABCG1, inhibiting the formation of foam cells and regulating the HDL level, to subsequently ameliorate AS. However, whether magnolol can ameliorate AS in a high fat-induced model or AS gene knock-out model requires further investigation.

\section{References}

1. Lozano R, Naghavi M, Foreman K, et al: Global and regiona mortality from 235 causes of death for 20 age groups in 1990 and 2010: A systematic analysis for the Global Burden of Disease Study 2010. Lancet 380: 2095-2128, 2012.

2. Vacca M, Degirolamo C, Mariani-Costantini R, Palasciano G and Moschetta A: Lipid-sensing nuclear receptors in the pathophysiology and treatment of the metabolic syndrome. Wiley Interdiscip Rev Syst Biol Med 3: 562-587, 2011.

3. Calkin AC and Tontonoz P: Transcriptional integration of metabolism by the nuclear sterol-activated receptors LXR and FXR. Nat Rev Mol Cell Biol 13: 213-224, 2012.

4. Chawla A, Boisvert WA, Lee $\mathrm{CH}$, et al: A PPAR gamma-LXR-ABCA1 pathway in macrophages is involved in cholesterol efflux and atherogenesis. Mol Cell 7: 161-171, 2001.

5. Venkateswaran A, Laffitte BA, Joseph SB, et al: Control of cellular cholesterol efflux by the nuclear oxysterol receptor LXR alpha. Proc Natl Acad Sci USA 97: 12097-12102, 2000.

6. Naik SU, Wang X, Da Silva JS, et al: Pharmacological activation of liver $\mathrm{X}$ receptors promotes reverse cholesterol transport in vivo. Circulation 113: 90-97, 2006.

7. Sun AL, Feng L and Liu RM: Preparative isolation and purification of honokiol and magnolol from Magnolia officinalis Rehd. et Wils by high-speed countercurrent chromatography. Fen Xi Hua Xue Bian Ji Bu 33: 1016-1018, 2005 (In Chinese).

8. Wang ZQ, Mi W, Liu XB, et al: The in vitro growth-inhibitory effect of Magnolia officinalis Rehd. et Wils. (MOR) on bacteria Shizhen Guo Yi Guo Yao 18: 2763, 2007 (In Chinese).

9. Zhang WW, Li Y, Wang XQ, et al: Effects of magnolol and honokiol derived from traditional Chinese herbal remedies on gastrointestinal movement. World J Gastroenterol 11: 4414-4418, 2005.
10. Ren SC, Fan YC and Li CC: Inhibitory effects of 30 kinds of Chinese herbal medicine on fungi in food. Adv Mater Res 343-344: 737-742, 2012.

11. Yang QH, Yang HW, Xie F and Zhang YP: Experimental study of Jiangzhi Ninggan capsules on rat model with fatty liver disease. Liaoning Zhong Yi Zazhi Bianji Bu 35: 1420-1422, 2008 (In Chinese).

12. Kotani H, Tanabe H, Mizukami H, Amagaya S and Inoue M: A naturally occurring rexinoid, honokiol, can serve as a regulator of various retinoid x receptor heterodimers. Biol Pharm Bull 35: $1-9,2012$.

13. Houck KA, Borchert KM, Hepler CD, Thomas JS, Bramlett KS, Michael LF and Burris TP: T0901317 is a dual LXR/FXR agonist. Mol Genet Metab 83: 184-187, 2004.

14. Chen CR, Tan R, Qu WM, et al: Magnolol, a major bioactive constituent of the bark of Magnolia officinalis, exerts antiepileptic effects via the GABA/benzodiazepine receptor complex in mice. Br J Pharmacol 164: 1534-1546, 2011.

15. Kumar S, Guru SK, Pathania AS, Kumar A, Bhushan S and Malik F: Autophagy triggered by magnolol derivative negatively regulates angiogenesis. Cell Death Dis 4: e889, 2013.

16. Zhang Y and Tang F: Advance in latest studies on pharmacological effects of magnolol. Zhongguo Zhong Yao Za Zhi 37: 3526-3530, 2012 (In Chinese).

17. Lee YJ, Lee YM, Lee CK, Jung JK, Han SB and Hong JT: Therapeutic applications of compounds in the Magnolia family. Pharmacol Ther 130: 157-176, 2011.

18. Vaughan AM and Oram JF: ABCA1 and ABCG1 or ABCG4 act sequentially to remove cellular cholesterol and generate cholesterol-rich HDL. J Lipid Res 47: 2433-2443, 2006.

19. Grefhorst A, Oosterveer MH, Brufau G, Boesjes M, Kuipers F and Groen AK: Pharmacological LXR activation reduces presence of SR-B1 in liver membranes contributing to LXR-mediated induction of HDL-cholesterol. Atherosclerosis 222: 382-389, 2012.

20. Wang $X$ and Paigen B: Genetics of variation in HDL cholesterol in humans and mice. Circ Res 96: 27-42, 2005.

21. Yasuda T, Grillot D, Billheimer JT, Briand F, Delerive P, Huet S and Rader DJ: Tissue-specific liver $\mathrm{X}$ receptor activation promotes macrophage reverse cholesterol transport in vivo. Arterioscler Thromb Vasc Biol 30: 781-786, 2010.

22. Linsel-Nitschke P and Tall AR: HDL as a target in the treatment of atherosclerotic cardiovascular disease. Nat Rev Drug Discov 4: 193-205, 2005.

23. Degoma EM and Rader DJ: Novel HDL-directed pharmacotherapeutic strategies. Nat Rev Cardiol 8: 266-277, 2011. 